\title{
ERRATUM
}

\section{PROJECTIVE MODULI FOR HITCHIN PAIRS}

\author{
[International Journal of Mathematics, Vol. 9, No. 1 (1998) 107-118] \\ Alexander Schimitt
}

The construction of the moduli space of Hitchin pairs in my paper Projective moduli for Hitchin pairs, Int. J. Math. Vol. 9, No. 1 (1998), 107-118, contains a removable error. Namely, the parameter space $\mathfrak{P}$ constructed in Sec. 4 is not the good one. Indeed, it parametrizes tuples $\left(\left[q: V \otimes \mathcal{O}_{X} \longrightarrow \mathcal{E}\right],[\varepsilon, f]\right)$ with $[q] \in \mathfrak{Q}$, $\varepsilon \in \mathbb{C}$, and $f: H^{0}(\mathcal{E}) \otimes H^{0}(\mathcal{E}(\nu)) \longrightarrow H^{0}(\mathcal{E}) \otimes H^{0}\left(\mathcal{O}_{X}(\nu)\right) \otimes H^{0}\left(\mathcal{O}_{X}(m)\right)$ some lifting of a homomorphism $\phi: \mathcal{E} \longrightarrow \mathcal{E} \otimes L$. The problem is that the equivalence relation coming from the $\mathrm{SL}(V)$-action on $\mathfrak{P}$ is not that of equivalence of Hitchin pairs, because different liftings of $\phi$ might not lie in the same orbit. Thus, Proposition 5.1 is false.

The correct parameter space $\mathfrak{R}$ is constructed in the obvious way as a closed subscheme of the following projective bundle $\widetilde{\mathfrak{R}}$ over the quot scheme $\mathfrak{Q}$ :

$$
\widetilde{\mathfrak{R}}:=\mathbb{P}\left(\mathcal{O}_{\mathfrak{Q}} \oplus \pi_{\mathfrak{Q} *}\left(\mathfrak{E}_{\mathfrak{Q}} \otimes \pi_{X}^{*} \mathcal{O}_{X}(\nu+m)\right)^{\vee} \otimes \pi_{\mathfrak{Q} *}\left(\mathfrak{E}_{\mathfrak{Q}} \otimes \pi_{X}^{*} \mathcal{O}_{X}(\nu)\right)\right) .
$$

One checks easily that, for the space $\mathfrak{R}$, the statement corresponding to Proposition 5.1 is correct. For any $a_{1}>a_{2}>0$, the line bundle $\mathfrak{L}_{\mathfrak{R}}^{\otimes a_{1}} \otimes \mathfrak{N}_{\mathfrak{P}}^{\otimes a_{2}}$ is very ample. Here, $\mathfrak{L}_{\mathfrak{R}}$ is the pullback of the very ample line bundle on $\mathfrak{Q}$ coming from the Pluecker embedding and $\mathfrak{N}_{\mathfrak{R}}$ the restriction of the tautological line bundle on $\widetilde{\mathfrak{R}}$. The computation of the stable points in $\mathfrak{R}$ w.r.t. such a line bundle corresponds to the computation of the stable points in $\mathfrak{P}$ w.r.t. $\mathcal{O}\left(a_{1}, a_{2}\right)$. However, we are no longer entitled to make the generous assumption in line $9-10$ on page 113 . Nevertheless, using a few extra arguments which are already present in our paper show that the argumentation still goes through. In particular, the main theorem of our paper remains unaffected. We don't give further details, because they are contained - together with generalizations of the results - in my forthcoming paper Framed Hitchin pairs which will appear in the Revue Roumaine de Mathématiques Pures et Appliquées.

Over curves, the construction is also contained in the general results of the author's preprint A universal construction for moduli spaces of decorated vector bundles over curves. 